recommended in LDLT using reduced LLS for small babies, especially when the graft has multiple hepatic veins.

$$
\begin{aligned}
& \text { Seisuke Sakamoto }{ }^{1} \\
& \text { Takanobu Shigeta }^{1} \\
& \text { Ikumi Hamano }^{1} \\
& \text { Akinari Fukuda }{ }^{1} \\
& \text { Toshihiko Kakiuchi }{ }^{1} \\
& \text { Naoto Matsuno }{ }^{1} \\
& \text { Hideaki Tanaka² } \\
& \text { Atsuko Nakagawa }{ }^{3} \\
& \text { Mureo Kasahara }{ }^{1}
\end{aligned}
$$

${ }^{1}$ Division of Transplant Surgery National Center for Child Health and

${ }^{2}$ Division of Pediatric Surgery National Center for Child Health and National Center for Child Health and
This work was supported, in part, by grants from the Scientific Research Fund of the Ministry of Education and by a research grant for immunology, allergy and organ transplant from the Ministry of Health, Labor and Welfare, Japan, and Foundation for Growth Science, Japan.

Address correspondence to: Seisuke Sakamoto, M.D., Ph.D., 2-10-1 Okura, Setagaya, Tokyo 157-8535, Japan.

E-mail: sakamoto-si@ncchd.go.jp

S.S. and M.K. participated in research design, data analysis, and writing of the manuscript; T.S., A.F., I.H., T.K., and A.N. participated in data analysis; and N.M. and H.T. participated in research design.

Received 4 October 2010.

Accepted 24 November 2010.

Copyright (C) 2011 by Lippincott Williams \& Wilkins

ISSN 0041-1337/11/9106-38

DOI: 10.1097/TP.0b013e3182088bb5

\section{REFERENCES}

1. Shirouzu Y, Kasahara M, Morioka D, et al. Vascular reconstruction and complications in living donor liver transplantation in in- fants weighing less than 6 kilograms: The Kyoto experience. Liver Transpl 2006; 12: 1224.

2. Kasahara M, Fukuda A, Yokoyama S, et al. Living donor liver transplantation with hyperreduced left lateral segments. J Pediatr Surg 2008; 43: 1575.

3. Sakamoto S, Egawa H, Kanazawa H, et al. Hepatic venous outflow obstruction in pediatric living donor liver transplantation using left-sided lobe grafts: Kyoto University experience. Liver Transpl 2010; 16: 1207.

4. Sakamoto S, Kasahara M, Shigeta T, et al. Feasibility of using the graft's umbilical vein as a patch graft for hepatic vein reconstruction in pediatric living donor liver transplantation. Transpl Int 2010; 23: 436.

5. Ueda M, Kozaki K. Surgical procedure for left lateral segmentectomy and left lobectomy. In: Tanaka K, Inomata Y, Kaihara S, eds. Living-donor liver transplantation: Surgical techniques and innovations. Barcelona, Prous Science 2003, pp 33.

6. Cheng YF, Chen CL, Haung TL, et al. Posttransplant changes of segment 4 after living related liver transplantation. Clin Transplant 1998; 12: 476.

\title{
Successful Heart and Liver Transplantation in a Swiss Patient With Glu89Lys Transthyretin Amyloidosis
}

Transthyretin (TTR) amyloidosis (MIM 176300) is the most common form of autosomal dominant hereditary systemic amyloidosis and causes familial amyloid polyneuropathy (FAP). TTR is a plasma transport protein for thyroid hormone and retinol-binding protein/ vitamin A. More than 100 distinct mutations have been described in the TTR gene, the Val30Met mutation being the most common. FAP is a worldwide disease with endemic foci in Portugal, Sweden, Japan, and Brazil. Significant phenotypic variability is associated with different mutations; it is also observed in patients harboring the same mutation (1). Initial presentation is usually a sensory peripheral polyneuropathy (PNP), followed by motor and autonomic impairment. Cardiac and renal dysfunction may be observed. Carpal tunnel syndrome (CTS) can be the primary clinical manifestation. Herein, we report an exceptional Glu89Lys TTR mutation in a Swiss family presenting with a severe phenotype characterized by unusually early heart dysfunction leading to heart and subsequent liver transplantation.

\section{CASE REPORT}

A 46-year-old Swiss woman was investigated for rapid-onset exertion- induced dyspnoea with generalized edema. She had a history of left CTS treated by median nerve release at the age of 42 years. Echocardiographic examination revealed hypertrophic cardiomyopathy, and subendocardial muscle biopsy revealed amyloid deposits (Fig. 1B) of the TTR type. Other investigations revealed no vitreous deposits, normal kidney function, and no paraproteinemia. Small interfascicular amyloid deposits were observed in a quadriceps muscle biopsy (Fig. 1C). As cardiac dysfunction was progressive, a heart transplant was successfully performed at the age of 49 years. Histologic examination of the explanted heart confirmed the presence of extensive amyloid deposits (Fig. 1D). After heart transplantation, the patient reported increasing pain and loss of sensation in the feet, slow bowel habit, and delayed urine flow. On examination, we found orthostatic hypotension, sensory loss, muscle weakness, and mild atrophy in the distal lower extremities with reduced Achilles tendon reflexes. Nerve conduction studies revealed a mild decrease of amplitude of motor and sensory action potentials and normal velocities except for bilateral slowing within the carpal tunnels. The sympathetic skin response to electrical stimuli was normal in the palms but not in the soles. The family history was in- formative with the patient's mother, maternal grandmother, and an uncle dying between the ages of 50 and 55 years because of cardiac dysfunction (Fig. 1A). An autopsy of the mother showed extensive amyloid deposits, prominently in the heart muscle. The patient's daughter had surgery for bilateral CTS at the age of 19 years with recurrence of sensory symptoms 4 years later. Direct DNA sequencing of the TTR gene showed a heterozygous Glu89Lys mutation in the proband and her daughter. We did not find any superimposed cause of axonal polyneuropathy, including diabetes, hypothyroidism, nutritional factors, rheumatologic or vasculitic conditions, M-protein, and infections. Therefore, the patient was treated with an orthotopic liver transplant (OLT) 18 months after the cardiac transplant and maintained on oral cyclosporine and mycophenolate mofetil. Although neurologic examination did not show obvious changes at 8 months post-OLT, nerve conduction studies parameters demonstrated minor worsening of the motor and sensory neuropathy. No amyloid resurgence was demonstrated in the transplanted heart using polarized light and congo-red staining on 23 successive endomyocardial biopsies. 

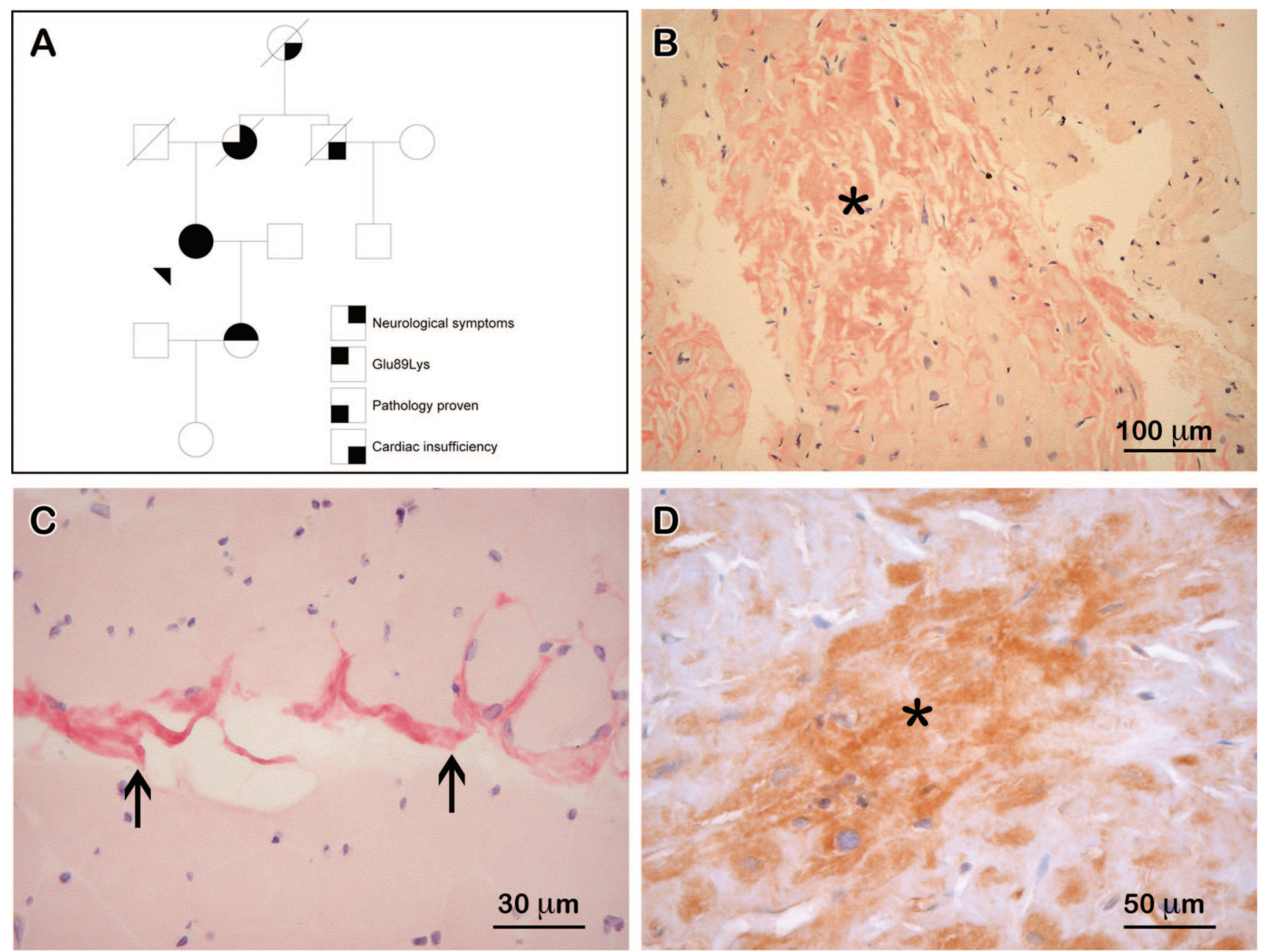

FIGURE 1. Family pedigree of Glu89Lys-mutated patients and results of tissue biopsies. (A) The index case appears with arrowhead; hallmarks are given for those family members with proven amyloidosis (autopsy or biopsy), Glu89Lys genotype, cardiac or neurologic manifestations. (B) Pretransplant subendocardial biopsy, demonstrating deposits of amyloid between cardiomyocytes; congo-red staining, asterisk. (C) Quadriceps muscle biopsy showing amyloid deposits between muscle fibers; congo-red staining, arrows. (D) Explanted heart showing marked interstitial amyloid deposits; transthyretin immunostaining, asterisk.

\section{DISCUSSION}

Our patient presented dramatically with a previously unrecognized FAP complicated by heart failure requiring heart transplantation. The neurologic disorder was identified late, based on history of CTS, painful PNP, and dysautonomic features. In the only previously reported Glu89Lys TTR FAP patient (an unrelated American patient), presentation was with an early painful PNP with hypertrophic cardiomyopathy that developed 6 years later, eventually leading to combined heart and liver transplants (2). According to the FAP World Transplant Registry, among 575 reported transplants, only 6 were combined liver and heart transplants (3). Cardiovascular symptoms are reported to be less responsive to OLT and account for 39\% of post-OLT deaths, mainly due to progressive amyloid heart deposits of wild-type TTR from the donor liver (4). Unlike the reported stabilization or improvement $(3,4)$, the patient's neuropathy slightly worsened after OLT. The cause of this worsening remains uncertain given that the patient developed concomitant steroid-induced diabetes. We found a high phenotypic penetrance in this Glu89Lys family with an onset between the ages of 20 and 45 years, but contrary to Val30Met FAP, we failed to find anticipation with maternal inheritance (5). Our re- port underlines the importance of TTR mutation screening in FAP to ensure adequate patient follow-up and for prognostic purposes, especially in relation to cardiac involvement with the Glu89Lys mutation. Regular cardiac evaluations will be undertaken for the youngest carrier who is in good health at the present time.

\section{ACIKNOWLEDGIMENT}

The authors thank Professors Richard Frackowiak and Manuel Pascual for critically reviewing this article.

Julien Niederhauser ${ }^{1}$ Johannes A. Lobrinus ${ }^{2}$ François Ochsner ${ }^{1}$ 
Christian Wider ${ }^{1}$ Florence Fellmann ${ }^{3}$ Patrick Yerly ${ }^{4}$ Anca T. Antonino ${ }^{5,6}$ Maria João Saraiva ${ }^{7}$ Darius Moradpour ${ }^{5,6}$ Thierry Kuntzer ${ }^{1}$ ${ }^{1}$ Neurology

Department of Clinical Neurosciences Lausanne University Hospital (CHUV)

Lausanne, Switzerland

${ }^{2}$ Pathology

Geneva University Hospital (HUG) Geneva, Switzerland

${ }^{3}$ Medical Genetics

Lausanne University Hospital (CHUV) Lausanne, Switzerland ${ }^{4}$ Cardiology

Lausanne University Hospital (CHUV)

Lausanne, Switzerland

${ }^{5}$ Gastroenterology and Hepatology Lausanne University Hospital (CHUV)

Lausanne, Switzerland

\author{
${ }^{6}$ Transplantation Center \\ Lausanne University Hospital (CHUV) \\ Lausanne, Switzerland \\ ${ }^{7}$ Molecular and Cellular Biology Institute \\ University of Porto \\ Porto, Portugal
}

The authors declare no conflict of interest.

Address correspondence to: Julien Niederhauser, M.D., Service de Neurologie, CHUV BH07, 1011 Lausanne, Switzerland.

E-mail: Julien.Niederhauser@chuv.ch

J.N., D.M., and T.K. participated in writing of the manuscript, data collection and analysis, and direct care of patient; J.A.L. participated in performing and commenting the histopathology section; F.O., P.Y., and A.T.A. participated in direct care of patient; C.W. participated in writing of the manuscript; and F.F. and M.J.S performed the genetic analyses.

Received 25 October 2010.

Accepted 22 November 2010.

Copyright (C) 2011 by Lippincott Williams \& Wilkins

ISSN 0041-1337/11/9106-40

DOI: 10.1097/TP.0b013e318208c0a2

\section{REFERENCES}

1. Benson MD, Kincaid JC. The molecular biology and clinical features of amyloid neuropathy. Muscle Nerve 2007; 36: 411.

2. Nakamura M, Hamidi AK, Benson MD. A novel variant of transthyretin (Glu89Lys) associated with familial amyloidotic polyneuropathy. Amyloid 2000; 7: 46.

3. Herlenius G, Wilczek HE, Larsson M, et al. Ten years of international experience with liver transplantation for familial amyloidotic polyneuropathy: Results from the Familial Amyloidotic Polyneuropathy World Transplant Registry. Transplantation 2004; 77: 64 .

4. Stangou AJ, Hawkins PN, Heaton ND, et al. Progressive cardiac amyloidosis following liver transplantation for familial amyloid polyneuropathy: Implications for amyloid fibrillogenesis. Transplantation 1998; 66: 229.

5. Hellman U, Alarcon F, Lundgren HE, et al. Heterogeneity of penetrance in familial amyloid polyneuropathy, ATTR Val30Met, in the Swedish population. Amyloid 2008; 15: 181.

\section{Maximizing Use of Marginal Liver Grafts for Transplantation: A Case Report of Absent Donor Extrahepatic Portal Vein}

Absence of the portal vein because of atrophy, agenesis, or aplasia can lead to both end-stage liver disease and hepatic tumors and therefore can be an indication for transplantation. We report the case of a successfully transplanted graft from a recipient with absence of the extrahepatic portal venous system. This situation has never been previously described in the literature.

\section{CASE REPORT}

This donor, deceased after brain death, was a 45 -year-old woman who died from a subarachnoid hemorrhage. Her medical history included a splenectomy at the age of 10 years (reason not known), an aneurysm clipped at the age of 15 years, and a laparotomy at the age of 21 years for a traumatic perforated stomach and intestine after a road traffic accident. She also had undergone a caesarian section at the age of 32 years after which she was jaundiced and a cholecystectomy at the age of 42 years. Just before organ donation, her liver function tests were normal.

The liver appearance was normal. However, during liver retrieval, it was noted that the extrahepatic portal vein was not present. A large-diameter common hepatic artery was identified. No difficulty was encountered in dissecting the porta because of collaterals. The pancreas was not retrieved.

Back-table dissection of the liver confirmed normal anatomy except for the absence of the extrahepatic portal vein to the level of the hilar plate. Portal vein collaterals were found and could be traced to a bifurcation into the left and right intrahepatic portal venous systems. Donor iliac vein was anastomosed to the intrahepatic portal vein bifurcation on the back table, and an angiogram was performed on the bench (Fig. 1a).

The recipient was a 68 -year-old woman with primary biliary cirrhosis. Model for end-stage liver disease score was18. The portal vein was patent. The donor portal venous anomaly and the absence of guiding medical literature were explained to the patient. After this, the patient elected to undergo transplantation.

The allograft was implanted using a side-to-side cavocavostomy with temporary portocaval shunt. Initial reperfusion followed arterial reconstruction. Reperfusion was tolerated, and liver appearance was satisfactory. The reconstituted donor vein was anastomosed in an end-to-end fashion to the patent recipient portal vein. A duct-to-duct biliary anastomosis was performed.
A postoperative computed tomography scan with vascular reconstruction demonstrates the transplanted hepatic vascular anatomy (Fig. 1b). Her alanine aminotransferase peaked at 1082 on day 2 and decreased to 155 by day 10 . However, alanine aminotransferase began to increase leading to a liver biopsy on day 13. This demonstrated acute cellular rejection, treated with methylprednisolone. The recipient has now been followed up for 4 years, and computed tomography scan confirms patency of the portal vein. Recurrent PBC has been confirmed by liver biopsy although she remains well with good liver function.

\section{DISCUSSION}

There are no previous reports of a liver with portal vein atrophy being transplanted. A possible explanation for the absence of an extrahepatic portal system seen in this donor is previous portal vein thrombosis. This is the likely explanation given her medical history.

Alternatively, it could be due to a true congenital abnormality. This is a rare condition in which the enteric blood bypasses the liver and drains directly into the systemic circulation. It can be subdivided into type 1 with no portal vein perfusion to the liver and type 2 where there remains a degree of 